\title{
When is Good, Good Enough? Methodological Pragmatism for Sustainable Guideline Development: Democratization of Processes for Setting Methodological Standards for Trustworthy Clinical Practice Guidelines \\ George P Browman ${ }^{1 *}$ and Melissa C Brouwers²
}

${ }^{1}$ School of Population and Public Health, University of British Columbia, Vancouver, BC, Canada

${ }^{2}$ Department of Oncology, McMaster University, Hamilton, ON, Canada

\section{Introduction}

The source paper contains two main messages in response to escalating demands for methodological rigor in the development and reporting of trustworthy clinical practice guidelines [1,2]. The messages are prompted by the experiences of the authors who have worked with, and advised individuals, groups and organizations committed to evidence-based methods in guideline development and research design.

In their advisory roles, the authors have noted consistent expressions of concern by clients who were skeptical about the marginal benefits associated with ever-increasing demands from the methodological community for more exact methods that were becoming impractical and expensive to implement in pursuit of trustworthy guidelines and related clinical research $[3,4]$. The authors of the paper have interpreted these client concerns as a backlash against increasing methodological dominance of the evidence-based movement that risks reversal, or erosion of the gains already made in promoting evidence-based processes in clinical research and care.

Among several other points made by the authors in support of the importance of methodological rigor in the development of guidelines, the two main messages relate to: 1 ) whether the additional costs, labor-intensiveness, and production delays for guidelines development to meet ever-increasing methodological standards of rigor in all circumstances are worth the resulting benefits; and 2) the appropriateness of the dominance of research methodologists in prescribing standards of rigor that affect so many other stakeholder groups in various ways.

The authors point out that pursuit of 'methodological purity' for trustworthy guidelines ignores the imperfections of real-world circumstances, and that formulaic approaches to defining what is methodologically acceptable hinders thoughtful consideration of issues within unique, real-world contexts [1].

The centre-piece of the paper is the description of a framework (the Efficiency-Validity Methodological Continuum) that is intended to facilitate negotiated thresholds of rigor among stakeholders in determining where the risks of compromise in methodological rigor are worth improved efficiencies in guideline development. But, the framework itself is not the most important takeaway message of this paper; rather, it is the need to democratize the process for determining methodologically acceptable thresholds in which methodologists trade their roles as rule-makers for appropriate methodological standards, to mediators, or expert advisors in helping stakeholders come to negotiated solutions that balance the risks to validity of guidelines (and the potential clinical consequences) against the costs of their production to meet stakeholder needs. An important implication of this approach is that the negotiation would need to be context specific, especially in relation to stakeholder values, the risks of the clinical condition under consideration in a guideline, the characteristics of the alternative interventions available and the affordability of producing a guideline and following its recommendations within real-world settings.

In the context of a more democratic approach to setting methodological standards involving stakeholder inputs, methodologists become educators, facilitators and guardians of a process of checks and balances to minimize risks of too lax or too rigid recommendations within a local or regional context. The process is intended to allow for universal methodological standards to be taught and acknowledged, but molded to circumstances and to the values of the negotiating parties.

The use of the framework (presented as a negotiation "tool") by negotiating parties in different jurisdictions might produce different processes, but three important methodological improvements are likely to result: 1) greater transparency in how methodological choices are made; 2) improved reporting of guideline development methods, including the processes used and the compromises made; and 3) direct research inquiry that would allow a more explicit investigation of the implications of the methods chosen on the credibility of recommendations, costs, and acceptability of the final products.

Adopting a negotiation process managed by methodologists may at first seem awkward, add administrative complexities and costs, but as reports accumulate about the experiences of stakeholders, we may learn more about how to garner the trust of all of those affected by guideline recommendations, and steadily improve the efficiency of guideline development. Finally, involving stakeholders in such processes can disseminate methodological knowledge and insights beyond the methodological community through a participatory approach that can lead to improved adherence to guideline recommendations [5]. In this context, stakeholders' "trust" in guidelines can be based on a more sophisticated appreciation of the methods used, rather than on formulaic rules, or pronouncements of 'expert' judgments.

Whether democratization of the process for determining the appropriateness of negotiated methodological compromises is workable, or even acceptable to stakeholders, will not be known

*Corresponding author: George P Browman, School of Population and Public Health, University of British Columbia, Vancouver, BC, Canada, E-mail gbrowman@yahoo.com

Received September 01, 2016; Accepted September 23, 2016; Published September 30, 2016

Citation: Browman GP, Brouwers MC (2016) When is Good, Good Enough? Methodological Pragmatism for Sustainable Guideline Development: Democratization of Processes for Setting Methodological Standards for Trustworthy Clinical Practice Guidelines. J Oncol Transl Res 2: 110.

Copyright: @ 2016 Browman GP, et al. This is an open-access article distributed under the terms of the Creative Commons Attribution License, which permits unrestricted use, distribution, and reproduction in any medium, provided the original author and source are credited. 
Citation: Browman GP, Brouwers MC (2016) When is Good, Good Enough? Methodological Pragmatism for Sustainable Guideline Development: Democratization of Processes for Setting Methodological Standards for Trustworthy Clinical Practice Guidelines. J Oncol TransI Res 2: 110.

Page 2 of 2

until such processes are tested to produce empirical evidence; but, challenging the dominance of the methodological community in making rules that affect so many other stakeholder groups may be an important thrust for the future acceptance of evidence-based guidelines, as well as continuing investments in their production. Such negotiated processes for guideline development can also make more explicit for all stakeholders involved the rationale and historical lessons that have led to the pursuit of high levels of methodological rigor, while clarifying the legitimate, but sometimes misplaced logic behind resistance to implementing key methodological strategies.

The authors' experiences with clients seeking methodological assistance in guideline development suggest that more democratic participatory processes may serve to reverse erosion of confidence among many stakeholders in the utility of good methodology for guideline development and what some stakeholders see as an elitist approach to evidence-based guideline development.

\section{References}

1. Browman GP, Somerfield MR, Lyman GH, Brouwers MC (2015) When is good, good enough? Methodological pragmatism for sustainable guideline development. Implementation Science 10: 28.

2. Agency for Healthcare Research and Quality. Clinical Practice Guidelines We Can Trust, 2011

3. Kovacs FM, Urrútia G, Alarcón JD (2014) "Overviews" should meet the methodological standards of systematic reviews. European Spine Journal 23 480.

4. Kung J, Miller RR, Mackowiak PA (2012) Failure of clinical practice guidelines to meet institute of medicine standards: Two more decades of little, if any, progress. Arch Intern Med 172: 1628-1633.

5. Jagosh J, Bush PL, Salsberg J, Macaulay AC (2015) A realist evaluation of community-based participatory research: Partnership synergy, trust building and related ripple effects. BMC Public Health 15: 725. 